\section{Modelo de investigación en gestión de proyectos para la investigación en ingeniería}

ECHA DE RECEPCIÓN: 8 de marzo FECHA DE APROBACIÓN: abril 20 Pp. 54-71

\section{Luis Alberto Esteban Villamizar* William Mauricio Rojas Contreras** Maritza del Pilar Sánchez Delgado***}

Research model in project management for engineering research

Madèles d'investigation en gestion de projets pour la recherche en ingénierie.

Madela de pesquisa em gestão de projetos para investigą̧ão em engenharia

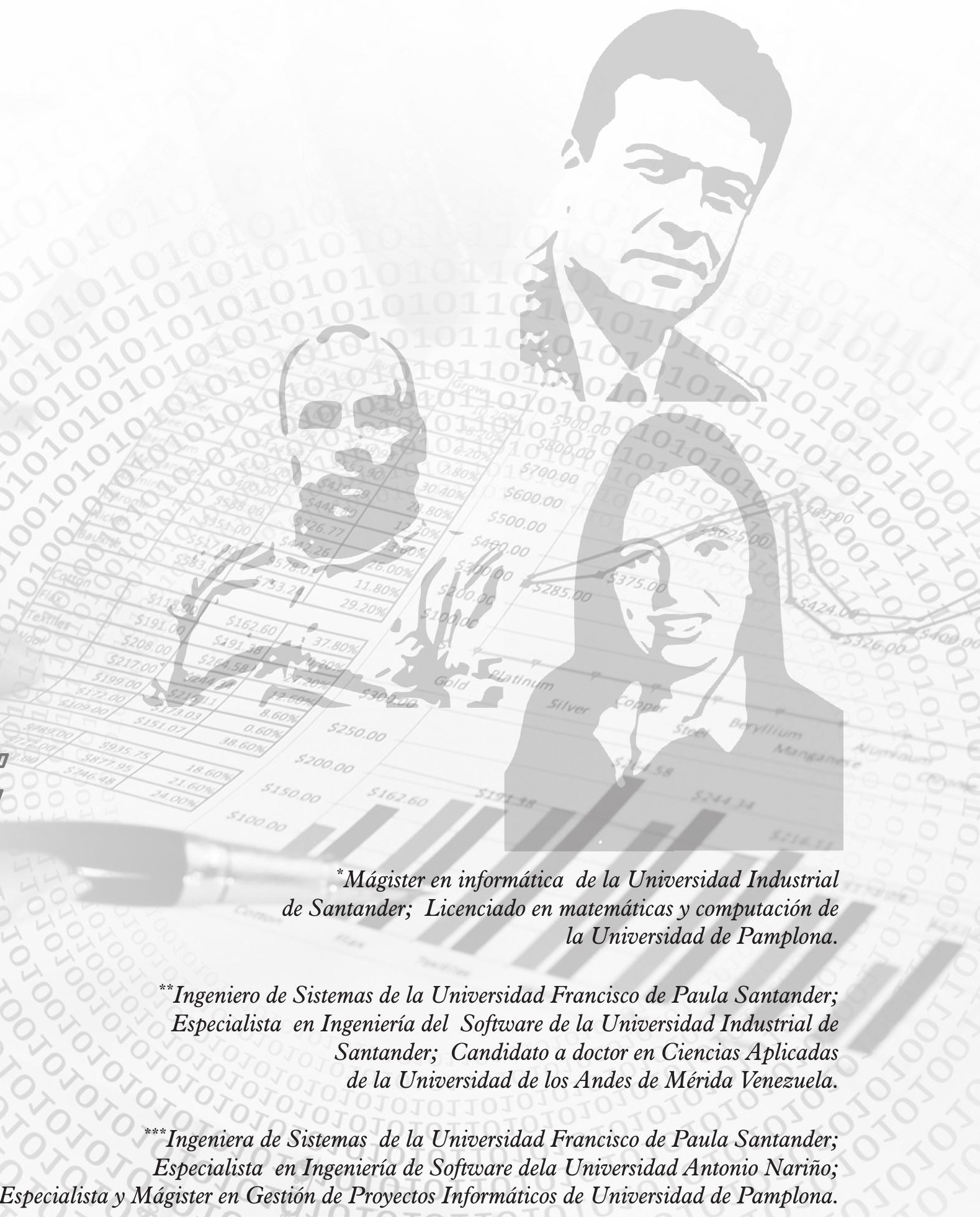




\section{RESUMEN}

Este artículo define un modelo de investigación en el área de gestión de proyectos adaptado dentro de los procesos de investigación en ingeniería, principalmente de la ingeniería del Software, con base en la guía de los fundamentos para la dirección de proyectos (Guía del PMBOK ${ }^{\circledR}$ ) y las Bases para las Competencias (NCB) de la Asociación Internacional de Dirección de Proyectos (IPMA).

\section{ABSTRACT}

This article shows a research model in the field of project management adapted from engineering research processes, mainly from software engineering, based on conceptual guidelines of project management and the existing competence bases of the International Association of Project Management.

\section{RESUMÉÉ}

Cet article définit un modèle d'investigation dans le domaine de la gestion de projet adapté principalement aux processus de recherche en ingénierie informatique sur la base du guide des connaissances fondamentales de la gestion de projets (Guide du PMBOK®) et des Bases pour la Connaissance (NCB) de l'Association Internationale de la Gestion de Projets (IPMA)

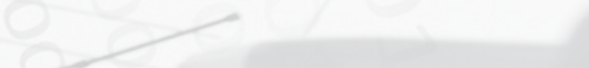

\section{RESUMO}

Este artigo define um modelo de investigação na área de gestão de projetos adaptado aos processos de investigação em engenharia, principalmente em engenharia de software, baseado na guia dos fundamentos para a direção de projetos (Guia do PMBOK®) e as Bases para as Competências (NCB) da Associação Internacional de Direção de Projetos (IPMA).

\section{Palabras claves}

Gestión de proyectos

Investigación en ingeniería del Software

Investigación en gestión de proyectos

Productos de ingeniería.

\section{Key words}

Project management

Software engineering research Project management research and engineering-based products.

\section{Mots clefs \\ Gestion de projets Recherche en ingénierie informatique \\ Recherche en gestion de projets \\ Produits de l'ingénierie.}

\section{(4) Palavras-chave \\ Gestão de projetos Investigação em engenharia de software Investigação em gestão de projetos Produtos de engenharia.}




\section{INTRODUCcIón}

E n Colombia, la gestión de proyectos se consideraba hace algunos años, como una competencia exclusiva de los profesionales en administración y áreas afines; sin embargo, cada vez son más los programas académicos de ingeniería que incluyen asignaturas de gestión de proyectos como parte de su proceso de formación, garantizando que el futuro profesional de la ingeniería esté dotado de competencias en la gestión de proyectos.

Paralelo a esta incursión de la gestión de proyectos como competencia de los ingenieros, se deben definir mecanismos de investigación, que aborden la gestión de proyectos como una línea más de investigación en cada ingeniería.
En este artículo se plantea una estrategia para incorporar la investigación en gestión de proyectos dentro de los procesos de investigación en ingeniería.

Está estructurado de la siguiente manera: en el apartado dos se describen los fundamentos teóricos claves en el estudio del modelo de investigación; en el apartado tres, se hace una descripción específica del modelo propuesto y un acercamiento a la incorporación del modelo a los procesos de investigación en gestión de proyectos; finalmente se presentan las conclusiones del trabajo.

\section{FUNDAMENTACIÓN TEÓRICA}

E n esta sección del artículo se presentan los conceptos fundamentales para justificar el modelo propuesto a partir de las competencias que deben tener los profesionales en ingeniería. Además, se exponen los modelos de investigación propios de la ingeniería del Software y las guías del cuerpo de conocimiento como elementos claves del modelo para la identificación de objetos de estudio en los procesos de investigación en gestión de proyectos.

\subsection{Competencias técnicas y metodológicas de los profesionales en ingeniería}

Existen diversas formas de clasificar las competencias que un determinado profesional debe tener para poder desempeñar sus funciones con ética, eficiencia y eficacia. Sin embargo, es muy común encontrar en dichas clasificaciones un grupo de habilidades que aquí llamaremos competencias técnicas y metodológicas para las ingenierías.

Es así como todos los programas de ingeniería deberían enfocar su esfuerzo en desarrollar estas habilidades para la construcción, operación y mantenimiento del producto objeto de ingeniería.

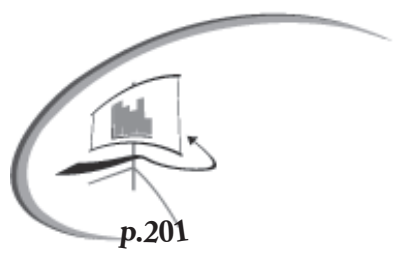


Figura 1. Competencias técnicas y metodológicas genéricas para la ingeniería

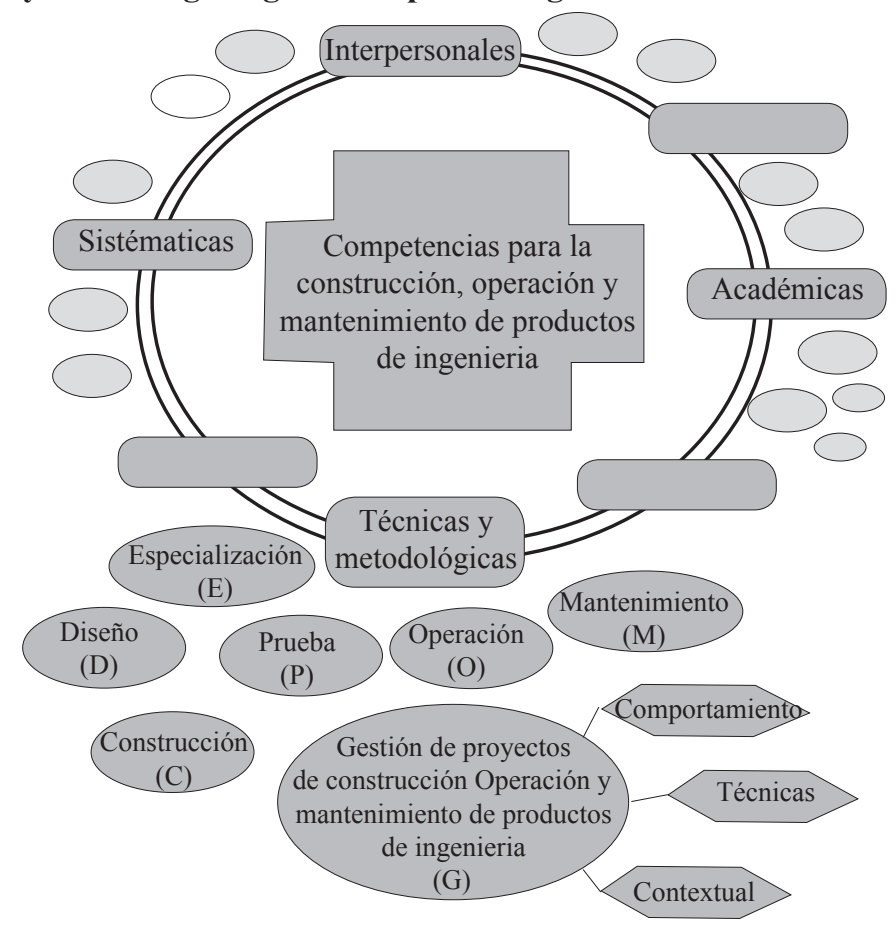

Fuente. Elaboración propia.

Las competencias técnicas y metodológicas para cualquier ingeniería pueden ser organizadas en sus áreas clave, como: Especificación de productos (E), Diseño (D), Construcción
(C), Prueba (P), Implantación (I), Operación (O), Mantenimiento $(\mathrm{M})$ y Gestión de proyectos $(\mathrm{G})$ de elaboración, operación y mantenimiento de dicho producto.

Se presenta a continuación el producto de algunas de estas ingenierías (tabla 1).

Tabla 1. Productos asociados a cada ingeniería

\begin{tabular}{|l|l|}
\hline \multicolumn{1}{|c|}{ Ingeniería } & Procesos de E, D, C, P, I, O, M y G aplicados a... \\
\hline Sistemas & Software o sistemas de información. \\
\hline Telecomunicaciones & Redes de comunicación. \\
\hline Eléctrica & Sistemas eléctricos. \\
\hline Electrónica & Sistemas electrónicos. \\
\hline Alimentos & Alimentos y proceso alimentarios. \\
\hline Civil & Obras de construcción (vías, edificios, etc.). \\
\hline Industrial & Procesos industriales. \\
\hline Química & Productos químicos. \\
\hline Petróleos & Procesos petroleros. \\
\hline Mecánica & Artefactos mecánicos (maquinarias). \\
\hline
\end{tabular}

Fuente. Elaboración propia. 
Estas habilidades son utilizadas por el profesional en ingeniería para realizar exactamente los mismos procesos utilizando sus propios métodos, herramientas y procedimientos existentes, ampliamente probados o experimentados para E, D, C, P, I, O, M y G del producto. Este último (la Gestión de proyectos -G) incluye habilidades en la dirección de proyectos como los propuestos por la International Project Management Association (IPMA), organizados a su vez en tres categorías: competencias de comportamiento, competencias contextuales y competencias técnicas (IPMA, 2009).

Es así como la investigación en una ingeniería se puede organizar de acuerdo con cada una de estas competencias, que constituirían líneas de investigación y cuyos productos de investigación serían el resultado de idear (inventar, innovar, adaptar) y validar dichas técnicas, métodos, herramientas y procedimientos.

Por lo tanto, se hace necesario definir un modelo de investigación cuyo producto aporte a la gestión de proyectos como disciplina de estudio adaptada a cada ingeniería en particular.

Tabla 2. Tipos de preguntas de investigación según Shaw

\begin{tabular}{|l|l|}
\hline \multicolumn{1}{|c|}{ Tipo de pregunta } & \multicolumn{1}{c|}{ Ejemplo } \\
\hline Método de desarrollo & $\begin{array}{l}\text { ¿De qué forma podemos hacer o desarrollar (o automatizar) X? } \\
\text { ¿Cómo mejoramos la forma de hacer o construir X? }\end{array}$ \\
\hline Método de análisis & $\begin{array}{l}\text { ¿Cómo podemos evaluar la calidad o corrección de X? } \\
\text { ¿Qué criterios permite elegir entre X o Y? }\end{array}$ \\
\hline $\begin{array}{l}\text { Diseño, evaluación o análisis de una } \\
\text { instancia particular }\end{array}$ & $\begin{array}{l}\text { ¿Cuál es el mejor diseño o implementación para X? } \\
\text { ¿Qué es la propiedad X del artefacto o método Y? } \\
\text { ¿Cómo podemos comparar X con Y? } \\
\text { ¿Cuál es el estado o práctica actual de X? }\end{array}$ \\
\hline Generalización, caracterización & $\begin{array}{l}\text { Dado X ¿qué será (necesariamente) Y? } \\
\text { ¿Qué significa exactamente X? }\end{array}$ \\
& ¿Cuáles son las características importantes de X? \\
¿Qué es un buen modelo formal o empírico para X? \\
¿Cuáles son las variedades de X? ¿Cómo relacionarlas?
\end{tabular}

Fuente. Elaboración a partir de Shaw (2002).

\footnotetext{
Fuente. Elaboración a partir de Shaw (2002).
}

\subsection{Modelo de investigación para adaptar a la investigación en gestión de proyectos}

En la ingeniería del Software, como disciplina, se pueden realizar procesos de investigación según los descritos por autores como Shaw (1990 y 2002), Galán y Cañate (2002), quienes explican cómo deben ser estos procesos mediante los tres componentes que caracterizan una investigación: el tipo de pregunta de investigación, el resultado y el método de validación utilizado para soportar el resultado (figura 2).

Figura 2. Tres aspectos que caracterizan una investigación

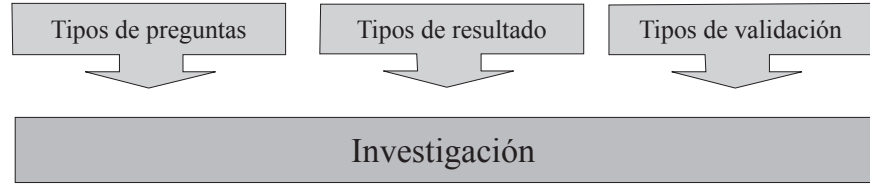

Fuente. Elaboración propia.

Los tipos de preguntas propios de la investigación en ingeniería del Software, según Shaw (2002), se ilustran en la tabla 2, en los cuales la variable $\mathrm{X}$ y la variable $\mathrm{Y}$, citadas en los ejemplos, son objetos de estudio propios de la ingeniería del Software. 
También es indispensable al inicio de cada investigación, determinar claramente el tipo de resultado o producto esperado. En la tabla 3 se muestran algunos tipos de resultados de investigación en ingeniería del Software según Shaw (2002).

Tabla 3. Tipos de resultados de investigación según Shaw

\begin{tabular}{|l|l|}
\hline \multicolumn{1}{|c|}{ Tipo de resultados } & \multicolumn{1}{c|}{ Ejemplo } \\
\hline Procedimiento o técnica & $\begin{array}{l}\text { Una forma nueva o mejor de hacer X, tal como diseño, imple- } \\
\text { mentación, mantenimiento, evaluación, selección de alternativas, } \\
\text { incluye técnicas operacionales para implementar, representar, } \\
\text { analizar, etc. }\end{array}$ \\
\hline $\begin{array}{l}\text { Método descriptivo o } \\
\text { cualitativo }\end{array}$ & $\begin{array}{l}\text { Estructuración de un problema (estilos, framework, patrones, } \\
\text { análisis de dominio no formales). } \\
\text { Generalizaciones informales bien argumentadas. Guías para inte- } \\
\text { grar resultados. }\end{array}$ \\
\hline Modelo empírico & Modelo predictivo basado en muestreos. \\
\hline Modelo analítico & $\begin{array}{l}\text { Modelo estructural suficientemente preciso para soportar análisis } \\
\text { formal o manipulación automática. }\end{array}$ \\
\hline Notación, herramienta & $\begin{array}{l}\text { Lenguaje formal para soportar técnicas o modelos (debe poseer } \\
\text { un cálculo, una semántica, etc.). } \\
\text { Herramienta que implementa una técnica. }\end{array}$ \\
\hline Solución particular & $\begin{array}{l}\text { Solución a un problema que usa principios de ingeniería y está } \\
\text { centrado más en el diseño que en la implementación. }\end{array}$ \\
\hline Respuesta, justificación & Resultado de un análisis específico, evaluación o comparación. \\
\hline Informe & Observaciones interesantes. \\
\hline
\end{tabular}

Fuente. Elaboración a partir de Shaw, (2002).

Estos productos de investigación, para el caso de la ingeniería del Software, podrían limitarse a métodos, herramientas y procedimientos según la definición de ingeniería del Software dada por uno de los más importantes autores en esta área, Roger Pressman: la Ingeniería del Software es una disciplina o área de la informática que ofrece método y técnicas para desarrollar y mantener Software de calidad (2010).
Los productos de una investigación deben ser validados dentro del mismo proceso de investigación, para lo cual Shaw (2002) realiza una síntesis de diferentes tipos de validación (tabla 4 ). 
Tabla 4. Tipos de validación de resultados de investigación según Shaw

\begin{tabular}{|l|l|}
\hline \multicolumn{1}{|c|}{ Tipo de validación } & \multicolumn{1}{|c|}{ Ejemplo } \\
\hline Análisis & $\begin{array}{l}\text { Hemos analizado nuestros resultados y los encontramos } \\
\text { satisfactorios mediante análisis formal (derivación rigurosa } \\
\text { y prueba), modelo empírico (experimento estadístico, uso } \\
\text { controlado de datos). }\end{array}$ \\
\hline Experiencia & $\begin{array}{l}\text { Mis resultados han sido usados sobre ejemplos reales por otras } \\
\text { personas y la evidencia de su corrección, utilidad o efectividad } \\
\text { es un modelo cuantitativo, un modelo empírico, datos basados } \\
\text { en la práctica, comparación con resultados similares actuales. }\end{array}$ \\
\hline Ejemplo & $\begin{array}{l}\text { Este es un ejemplo de cómo funciona; un ejemplo quizás, } \\
\text { motivado por la realidad o por un sistema en construcción. }\end{array}$ \\
\hline Evaluación & $\begin{array}{l}\text { Bajo los criterios establecidos, mis resultados describen (mo- } \\
\text { delo descriptivo) adecuadamente el fenómeno de interés, con- } \\
\text { tabilizan (modelo cualitativo) el fenómeno de interés, está } \\
\text { capacitado para predecir (modelo empírico) porque produce } \\
\text { resultados que coinciden con los datos reales. } \\
\text { Incluye estudios de factibilidad, proyectos pilotos, etc. }\end{array}$ \\
\hline Persuasión & $\begin{array}{l}\text { Hemos trabajado duro y con nuestra experiencia creemos...; } \\
\text { técnica) si usted lo hace de la siguiente forma...; (sistema) un } \\
\text { sistema construido como este podría...; (modelo) este modelo } \\
\text { parece razonable... } \\
\text { Si la pregunta original fuese sobre factibilidad, un sistema } \\
\text { funcionando, incluso sin análisis, puede ser persuasivo. }\end{array}$ \\
\hline No hay intentos serios de evaluar los resultados. \\
\hline
\end{tabular}

Fuente. Elaboración a partir de Shaw.

De esta forma, se puede considerar como pertinente una investigación en ingeniería del Software cuando se defina claramente el tipo de pregunta, el tipo de resultado y el tipo de validación del resultado según estas clasificaciones, y además cuando el objeto de estudio esté comprendido entre los límites del dominio de conocimiento para la ingeniería del Software. Para esto se puede usar la guía del SWEBOK ${ }^{\circledR}$ (IEEE,2004) como herramienta para seleccionar dicho objeto de estudio. Para el caso de la gestión de proyectos, es necesario estudiar otro cuerpo de conocimiento: la guía del $\mathrm{PMBOK}^{\circledR}$ (PMI, 2009).

\subsection{Guías para el cuerpo de conocimiento de una ciencia 0 disciplina}

El cuerpo de conocimiento de una ciencia o de una disciplina, está conformado por toda la información que se ha producido en ella; esto incluye libros, artículos, experiencias documentadas, entre otros, que de una u otra forma son publicados y aceptados como conocimiento válido.

Recopilar toda esta documentación no se ve como tarea fácil, pero algunas organizaciones mundiales han tratado de organizar los contenidos de dichas disciplinas en lo que se conoce como guías para el cuerpo de conocimiento (BOK). 
Figura 3. Interpretación de un cuerpo de conocimiento (Body Of Knowledgement - BOK)

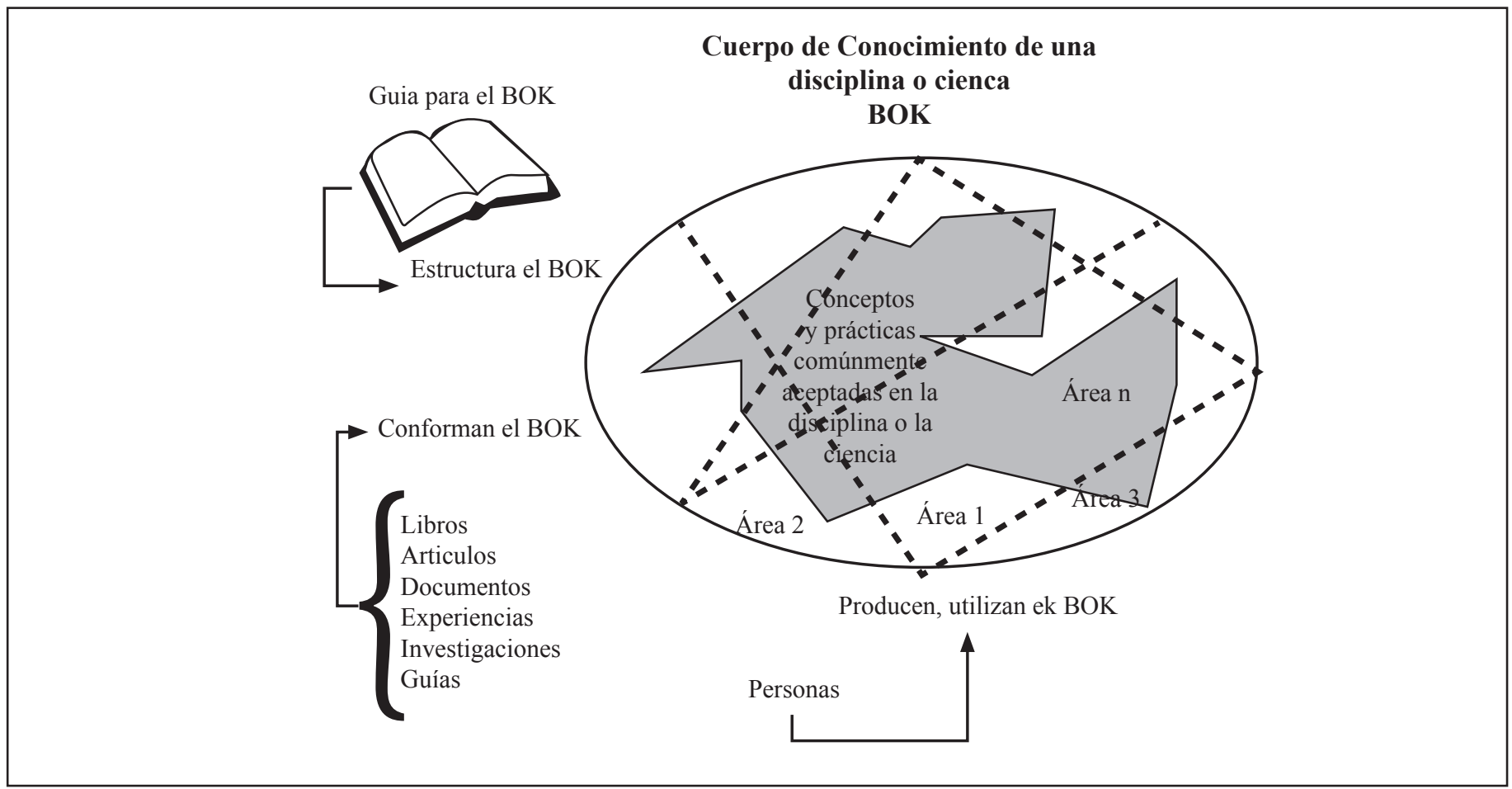

Fuente. Elaboración a partir de Body Of Knowledgement-Bok.

Estas guías proporcionan la información necesaria y suficiente para definir objetos de estudio dentro de procesos de investigación propios en cada disciplina.

Para el caso de la ingeniería del Software se dispone de la guía para el SWEBOK ${ }^{\circledR}$, Software Engineering Body of Knowledge (IEEE, 2004), la cual es un documento creado por la Software Engineering Coordinating Committee y promovido por la IEEE Computer Society. Ha sido un gran aporte para la profesionalización de ingeniería del Software.

Esta guía para el SWEBOK ${ }^{\circledR}$ fue elaborada con los siguientes objetivos (IEEE, 2004):

- Promover una vista consistente de la ingeniería del Software en todo el mundo.

- Clarificar el lugar y fijar los límites de la ingeniera del Software respecto a otras disciplinas tales como las ciencias computacionales, la gestión de proyectos, la ingeniería computacional y las matemáticas.

- Caracterizar los contenidos de la ingeniería del Software y suministrar un acceso temático al cuerpo de conocimiento de ella.

- Proveer los fundamentos para el desarrollo de currículos y para la certificación individual.

La guía del SWEBOK ${ }^{\circledR}$ organiza este cuerpo de conocimiento (de la ingeniería del Software) en diez áreas de conocimiento que pueden ser modelo para cualquier otra ingeniería que no disponga de una guía que la organice. Es así como esta se constituye en una fuente de objetos de estudio para los procesos de investigación en ingeniería del Software. 
Figura 4. Áreas de conocimiento para la ingeniería del software según el SWEBOK ${ }^{\circledR}$

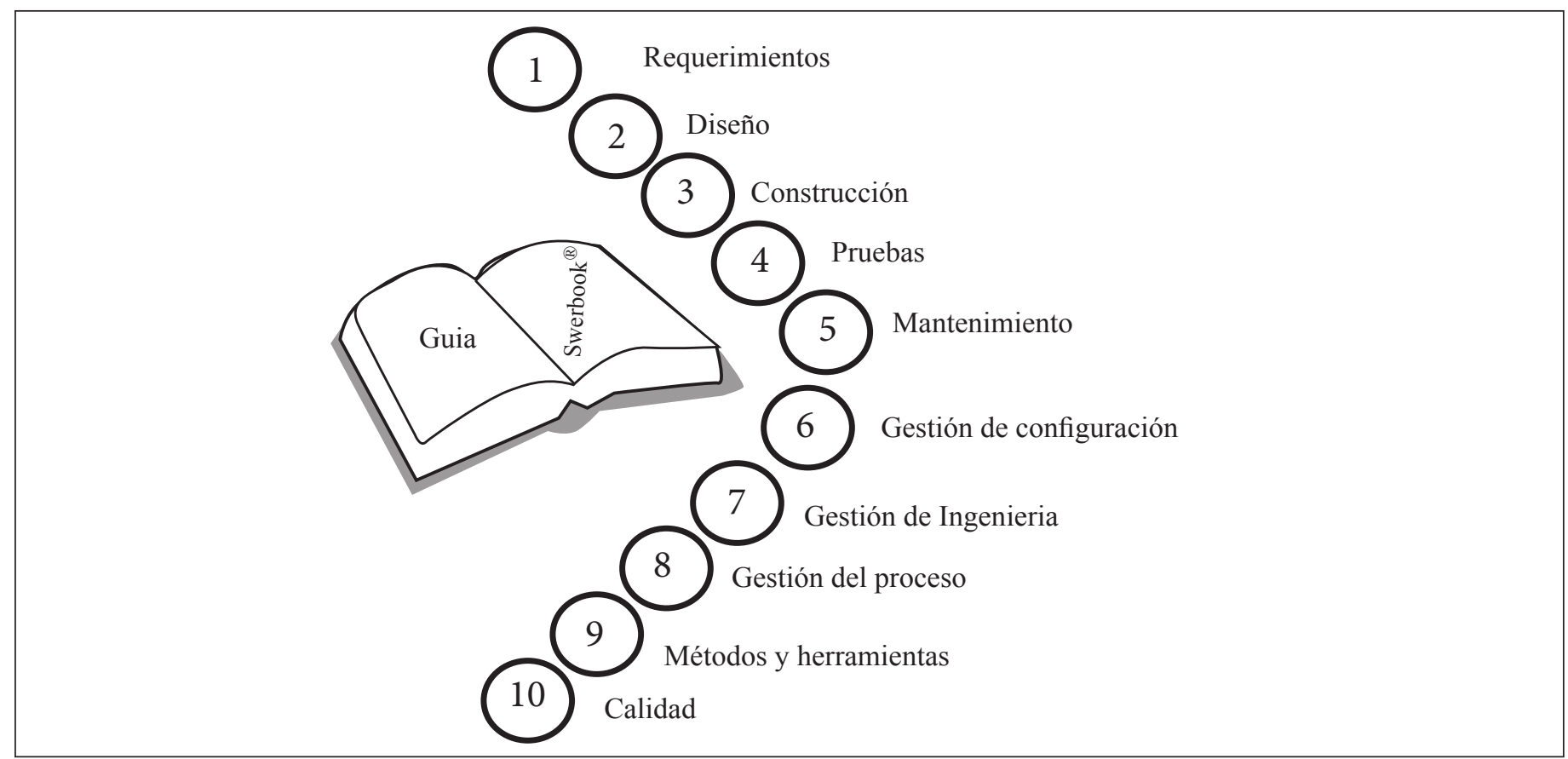

Fuente. SEWBOK ${ }^{\circledR}$

En la guía del $\mathrm{SEWBOK}^{\circledR}$, la gestión de proyectos de Software está incluida dentro del área de conocimiento de gestión de ingeniería.

Por otro lado, para el caso de la gestión de proyectos como disciplina, se puede utilizar la guía del $\mathrm{PMBOK}^{\circledR}$ (PMI, 2009) como fuente de objetos de estudio, en la medida en que ofrece métodos, herramientas y procesos para la gestión de proyectos de cualquier índole.

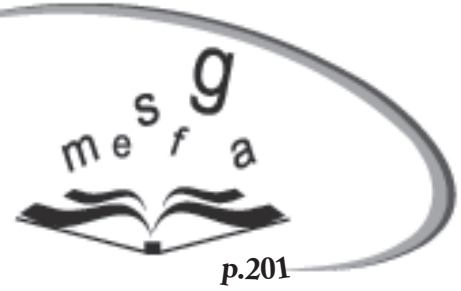

Por lo tanto una línea de investigación en el área de gestión de proyectos, dentro de una ingeniería, podría enfocar sus esfuerzos en adaptar, generar, innovar, diseñar métodos, herramientas $\mathrm{y}$ procedimientos debidamente validados y que puedan ser utilizados en proyectos propios de dicha inge-niería en particular.

\subsection{La guía del PMBOK ${ }^{\circledR}$}

La guía del PMBOK $^{\circledR}$, desarrollada por el Project Management Institute $\left(\mathrm{PMI}^{\circledR}\right)$ "es la norma para dirigir la mayoría de proyectos, la mayor parte del tiempo, en diversos tipos de industria. Esta norma describe los procesos, herramientas y técnicas utilizados para dirigir un proyecto con miras a un resultado exitoso" (PMI, 2009). Esta norma se ha convertido en un estándar para la dirección de proyectos y, a diferencia de otras guías, para el cuerpo de conocimiento, esta guía tiene dos enfoques (PMI, 2009), como se muestra en la figura 8. 
Figura 5. Los dos enfoques de la guía del PMBOK ${ }^{\circledR}$

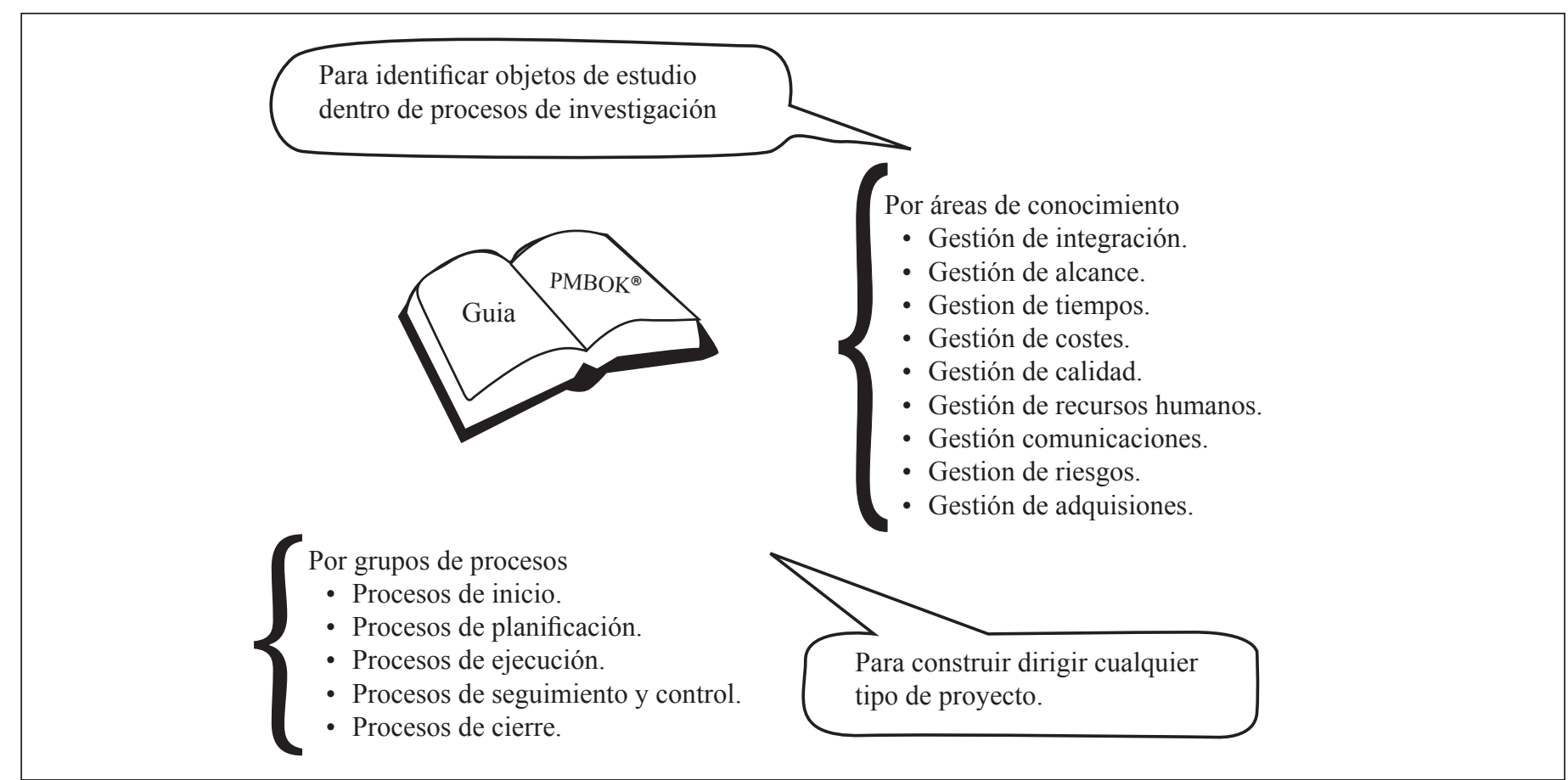

Fuente. Elaboración a partir de $\mathrm{PMBOK}^{\circledR}$

Si se desea utilizar esta guía como norma para la dirección de proyectos, es necesario realizar el estudio por grupos de procesos. Sin embargo, también brinda un acceso temático al cuerpo de conocimiento de la gestión de proyectos, para lo cual se debe estudiar por áreas de conocimiento (figura 6).

Figura 6. Las áreas de conocimiento de la gestión de proyectos según el PMBOK ${ }^{\circledR}$

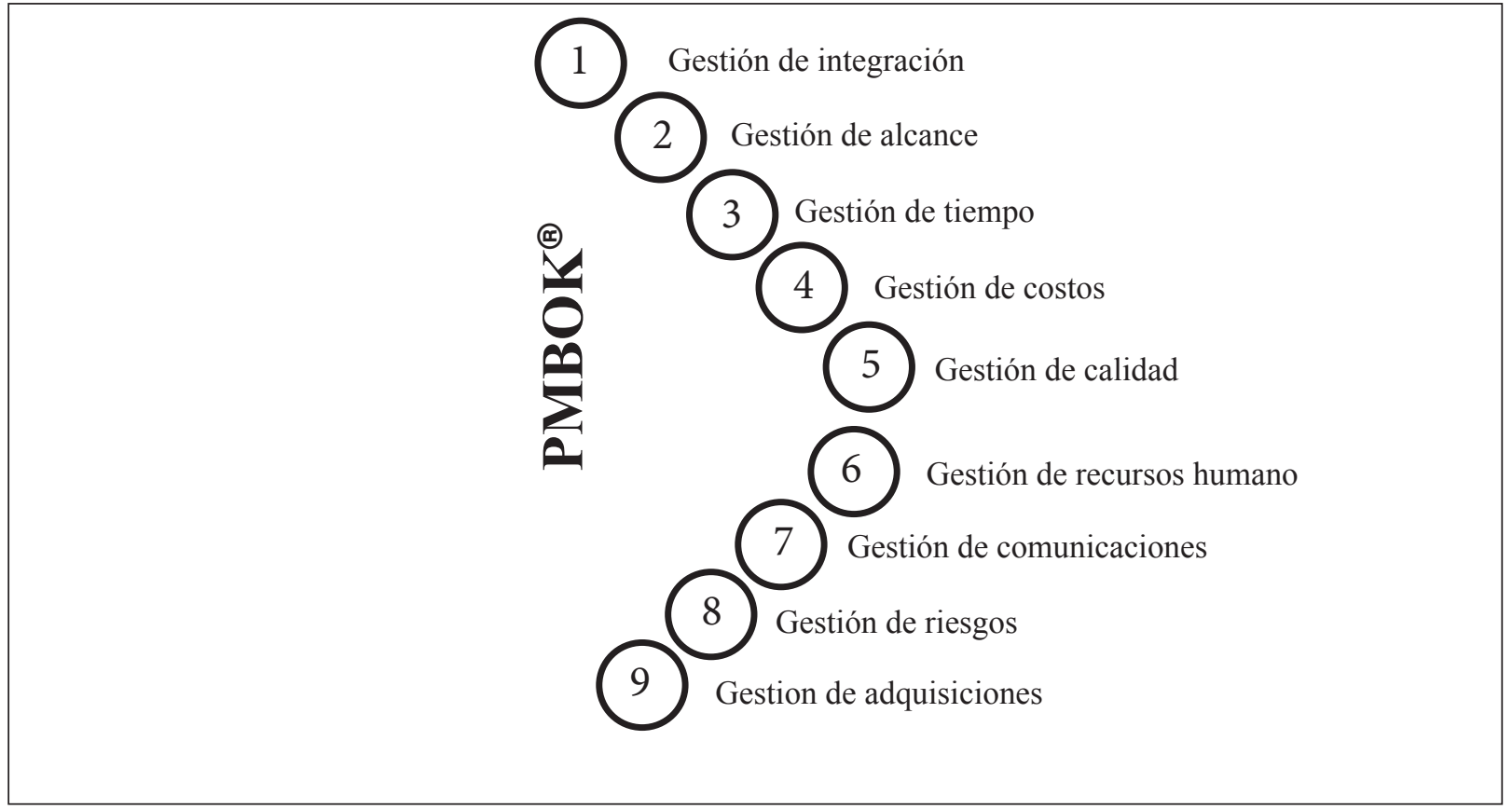

Fuente. Elaboración a partir de $\mathrm{PMBOK}^{\circledR}$ 
Cada una de estas áreas (PMI, 2009) se constituye también en fuente de objetos de estudio para proyectos de investigación en ingeniería, pues se pueden definir y validar métodos, técnicas, procedimientos propios de la gestión de proyectos de ingeniería.

Como norma para la dirección de proyectos es necesario aplicarla por grupos de procesos, de tal manera que toda actividad del proyecto pueda ser clasificada en dos grandes categorías (Rojas, 2011): actividades de gestión y actividades técnicas propias para la generación del producto único motivo del proyecto. A su vez, las actividades de gestión se organizan dentro de procesos propuestos por la guía del $\mathrm{PMBOK}^{\circledR}$ en cinco grupos de procesos: inicio, planificación, ejecución, seguimiento control y cierre.

Figura 7. Los grupos de procesos de gestión según la guía del PMBOK ${ }^{\circledast}$

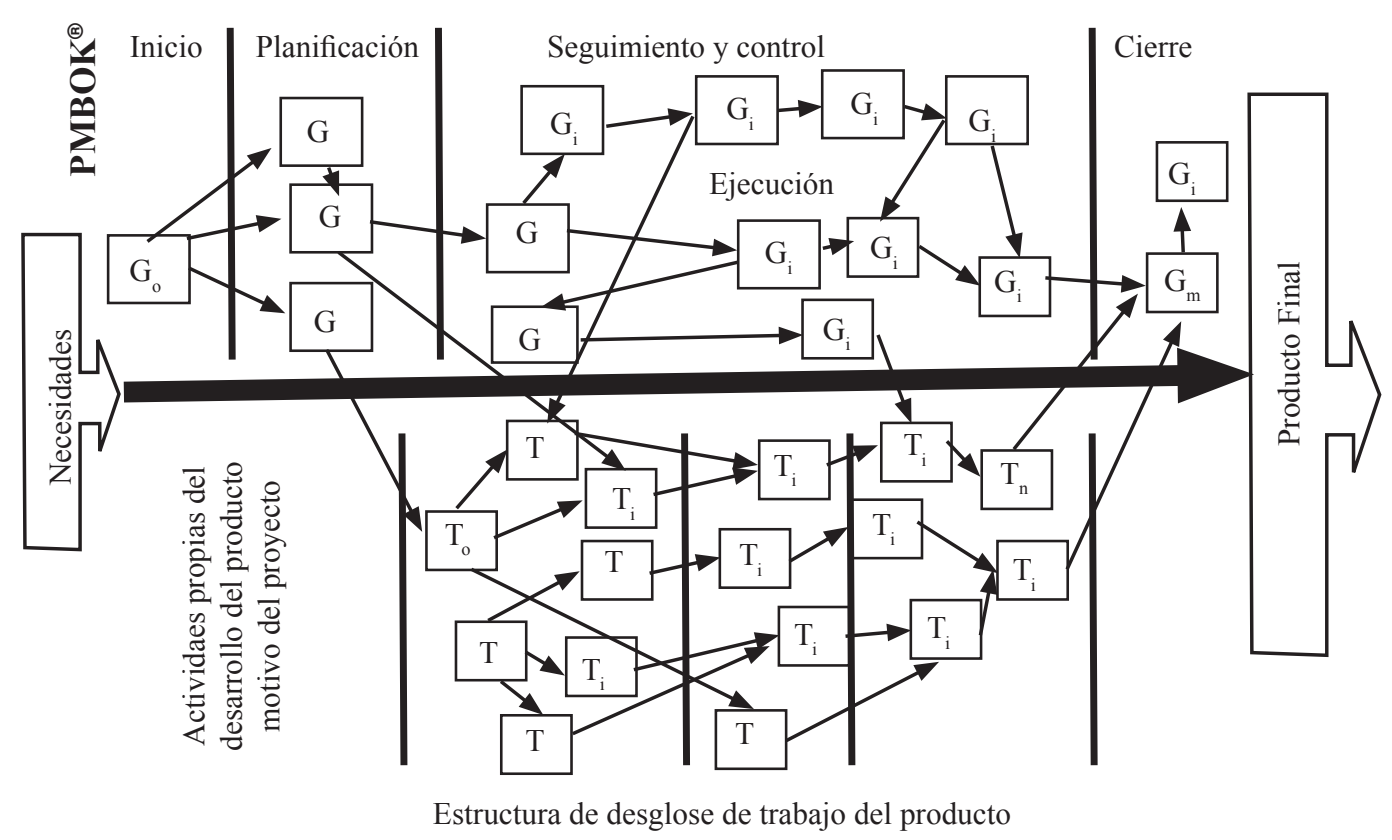

Fuente. Elaboración a partir de $\mathrm{PMBOK}^{\circledR}$.

Actualmente, se dispone de la versión 5 de la guía del $\mathrm{PMBOK}^{\circledR}$, que mantiene esta estructura de grupos de procesos pero que incrementó el número de procesos de 42 (en la versión 4) a 47 y separó algunos de estos procesos en una nueva área de conocimiento denominada "Gestión de Stakeholders" (PMI, 2013).
De igual manera el PMI ${ }^{\circledR}$, en conjunto con el IEEE CS, está trabajando en una extensión de la guía para proyectos de desarrollo de Software (PMI, 2013a).

Este artículo está basado en la versión 4 pero el modelo aquí propuesto puede ser adaptado a la nueva versión. 


\section{INVESTIGACIÓN EN GESTIÓN DE PROYECTOS}

Ne ilustra, (figura 8) a manera de procedimiento, el modelo $\checkmark$ propuesto en este artículo para abordar procesos de investigación en gestión de proyectos, adaptado del modelo de Shaw (2002).

Figura 8. Modelo propuesto para realizar procesos de investigación en gestión de proyectos

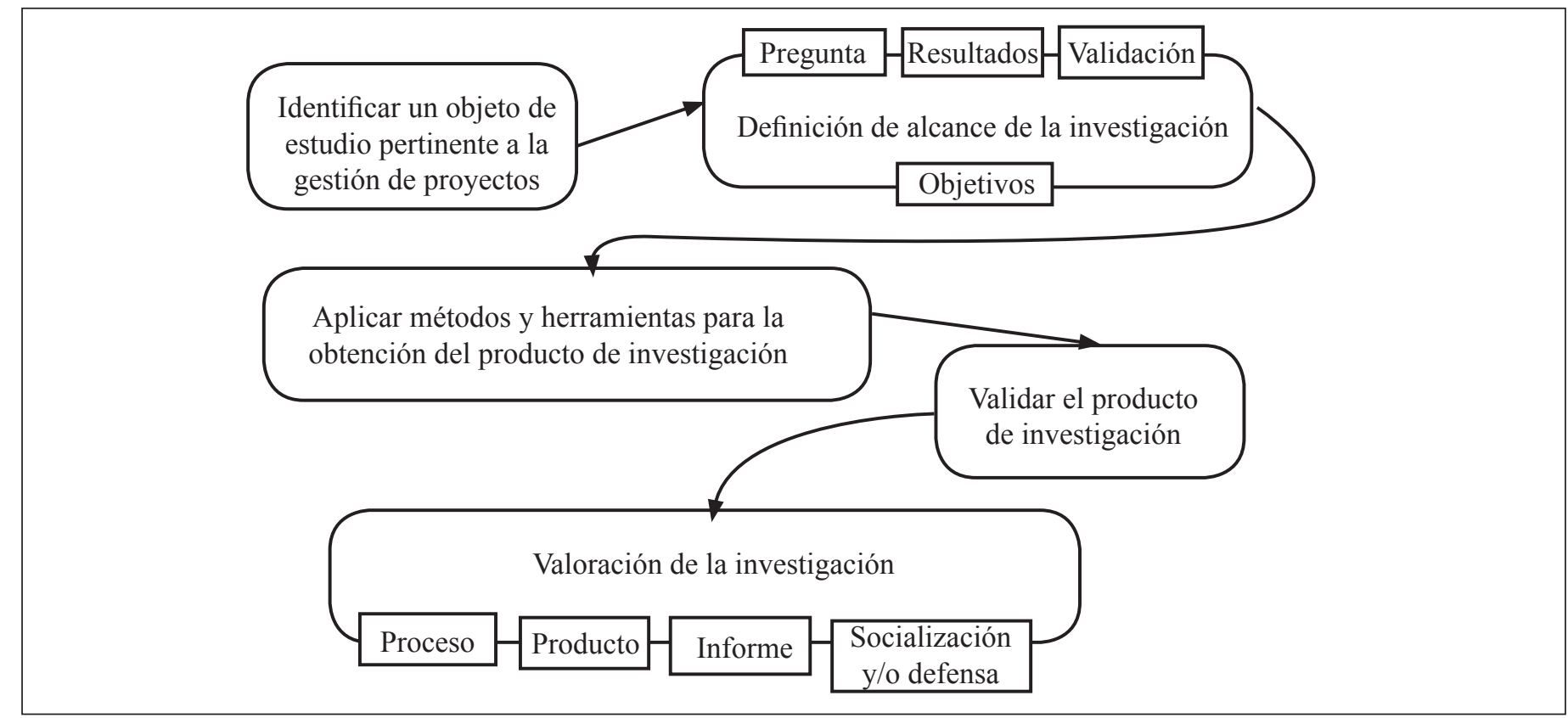

Fuente. Elaboración propia.

Es indispensable, como primer factor para el desarrollo de cualquier investigación, definir el cuerpo de conocimiento sobre el cual se está aportando (en este caso el cuerpo de conocimiento de la gestión de proyectos), dado que en algunos proyectos de investigación se aplica el saber de un cuerpo de conocimiento para aportar a un segundo cuerpo de conocimiento; por tal razón sería válido dicho producto de investigación en la segunda disciplina y no en la primera.

Por ejemplo, si se desarrolla un aplicativo Software (producto de ingeniería del Software) para ser puesto en marcha dentro de un proyecto en educación (es decir, que las variables objeto de estudio son de educación), se considera pertinente para el área de educación, mas no aporta ningún conocimiento al área de ingeniería del Software. Por lo tanto, es indispensable definir la pertinencia en términos del objeto de estudio, es decir, que si este pertenece al cuerpo de conocimiento de la gestión de proyectos, se puede considerar proyecto de investigación en gestión de proyectos, independientemente del tipo de proyecto sobre el que sea útil el producto de dicha investigación.

Dado que la gestión de proyectos es pertinente a cualquier ingeniería, se tendría la posibilidad de abordar objetos de estudio de la gestión de proyectos, teniendo como fuente las áreas de conocimiento del $\mathrm{PMBOK}^{\circledR}$, en las cuales se puedan plantear modelos, métodos, procedimientos y herramientas para alguno de los procesos dentro de las áreas de conocimiento de la gestión de proyectos. Entre ellos se tiene (PMI, 2009): 


\section{Gestión de la integración del proyecto}

- Desarrollar el mapa de navegación del proyecto.

- Desarrollar el plan para la dirección del proyecto.

- Dirigir y gestionar la ejecución del proyecto.

- Monitorear y controlar el trabajo del proyecto.

- Realizar el control integrado de cambios.

- Cerrar proyecto o fase.

\section{Gestión del alcance del proyecto}

- Recopilar requisitos.

- Definir el alcance.

- Crear EDT.

- Verificar el alcance.

- Controlar el alcance.

\section{Gestión del tiempo del proyecto}

- Definir las actividades.

- Secuenciar las actividades.

- Estimar los recursos de las actividades.

- Estimar la duración de las actividades.

- Desarrollar el cronograma.

- Controlar el cronograma.

\section{Gestión de los costos del proyecto}

- Estimar los costos.

- Determinar el presupuesto.

- Controlar los costos.

\section{Gestión de la calidad del proyecto}

- Planificar la calidad.

- Realizar el aseguramiento de calidad.

- Realizar el control de calidad.

\section{Gestión de los recursos humanos del proyecto}

- Desarrollar el plan de recursos humanos.

- Adquirir el equipo del proyecto.

- Desarrollar el equipo del proyecto.

- Dirigir el equipo del proyecto.

\section{Gestión de las comunicaciones del proyecto}

- Identificar a los interesados (Stakeholders).

- Planificar las comunicaciones.

- Distribuir la información.

- Gestionar las expectativas de los interesados.

- Informar el desempeño.

\section{Gestión de los riesgos del proyecto}

- Planificar la gestión de riesgos.

- Identificar los riesgos.

- Realizar el análisis cualitativo de riesgos.

- Realizar el análisis cuantitativo de riesgos.

- Planificar la respuesta a los riesgos.

- Monitorear y controlar los riesgos.

\section{Gestión de las adquisiciones del proyecto}

- Planificar las adquisiciones

- Efectuar las adquisiciones

- Administrar las adquisiciones

- Cerrar las adquisiciones

Sobre cada uno de estos procesos, se consideraría pertinente el desarrollo de modelos, métodos, procedimientos y herramientas como productos de investigación validados dentro del mismo proceso de investigación. Esto permite identificar infinidad de objetos de investigación, susceptibles de procesar mediante el modelo aquí propuesto. 
Figura 9. Posibles productos de investigación vs. áreas de conocimiento de la gestión de proyectos

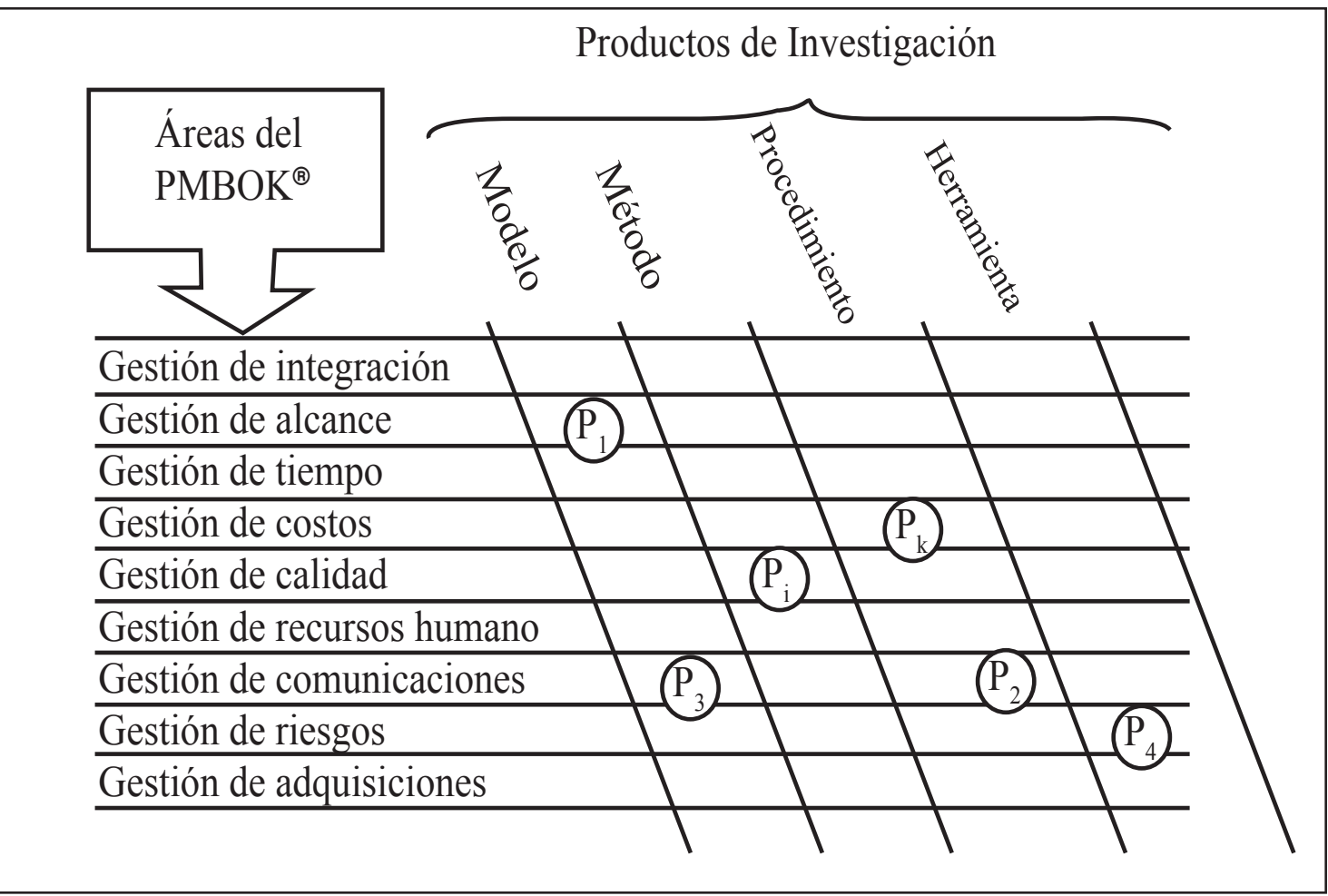

Fuente. Elaboración propia.

Una vez seleccionado el objeto de estudio y el producto de investigación, se podría recurrir a una especie de patrón en el diseño de los propósitos del trabajo de investigación, que se podrían sintetizar en tres objetivos:

- Realizar un estado del arte sobre el objeto de estudio.

- Definir el modelo, método, procedimiento o herramienta producto de la investigación.

- Validar la utilidad de dicho producto de investigación.

El primer objetivo esta encaminado a conocer el origen, evolución y perspectiva futura de los estudios realizados sobre dicho objeto. El segundo, se constituye en la tesis de dicha investigación y el tercero, mide o justifica la validez de la tesis.

Para dar cumplimiento al segundo objetivo es necesaria la definición de hipótesis demostrables por medio de diferentes métodos, tanto empíricos como teóricos, teniendo en cuenta el tipo de investigación, como lo indican Pinzón y Ospina (2006).

Finalmente, el producto de investigación en gestión de proyectos aplicados a una ingeniería en particular, debe ser valorado según los siguientes factores (figura 10). 
Figura 10. Estructura del proceso de valoración de resultados de investigación

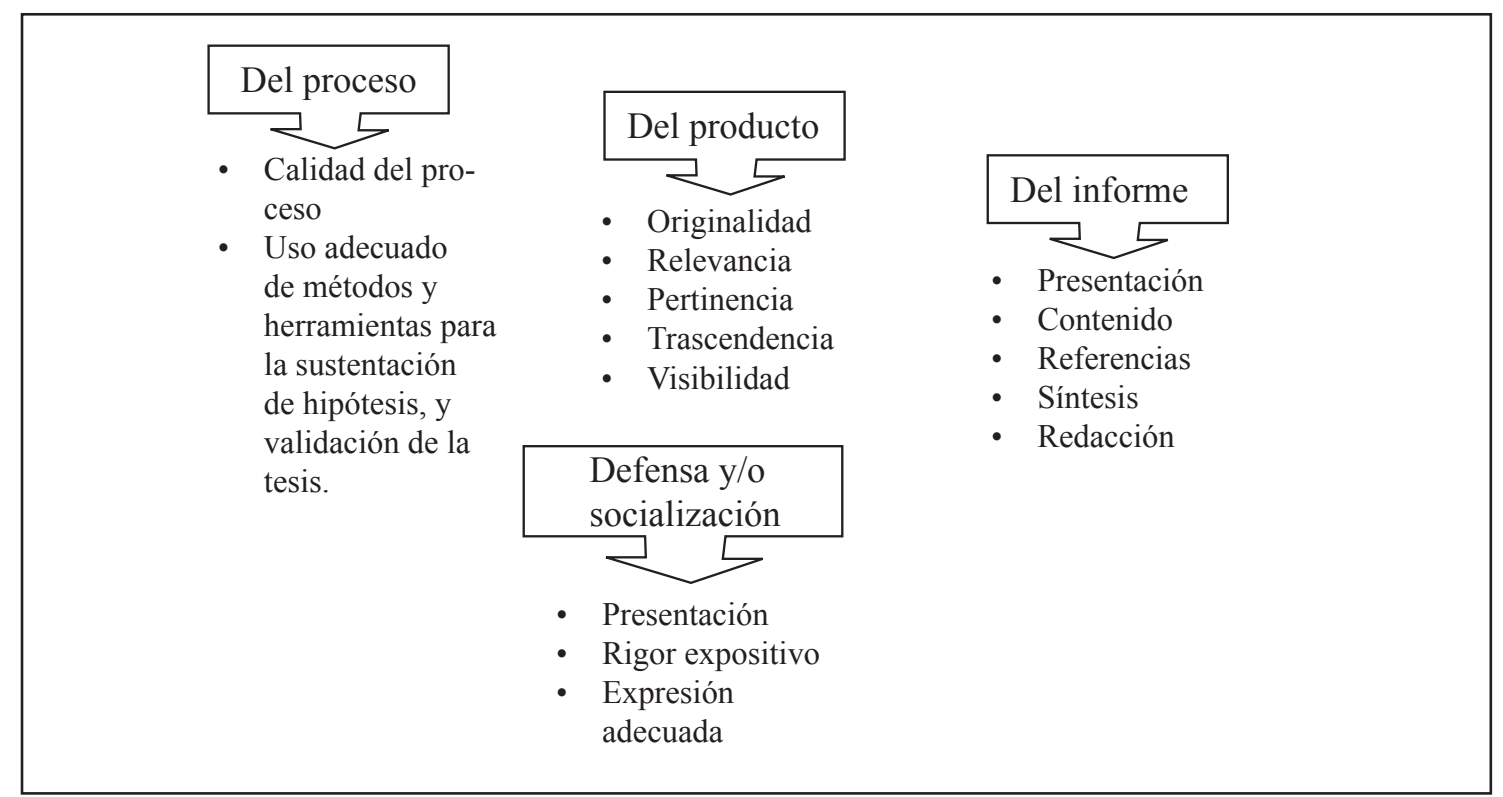

Fuente. Elaboración propia.

\subsection{Valoración del proceso de investigación}

Los siguientes criterios sobre el proceso de investigación se pueden tener en cuenta en el momento de la evaluación:

- Calidad del proceso: solidez científica, técnica o metodológica del trabajo de investigación. Exactitud de los resultados, pruebas o reflexiones.

- Uso adecuado de métodos y herramientas para la sustentación de hipótesis y validación de la tesis.

\subsection{Valoración del producto de investigación}

El principal producto de investigación se refiere al conocimiento aportado por la investigación en el cuerpo de conocimiento pertinente a la disciplina de la ingeniería, incluyendo la gestión de proyectos.

Para el caso de una ingeniería en particular, los aportes de conocimiento deben estar relacionados con la guía del $\mathrm{PMBOK}^{\circledR}$ adaptada a procesos de desarrollo (construcción), operación o mantenimiento del producto propio de dicha ingeniería.
Los criterios a tener en cuenta son:

- Originalidad: conocimiento no socializado previamente, o experiencia no reportada antes. Una técnica o método no usado anteriormente. Se puede resolver intentando dar respuesta a interrogantes como ¿Ha sido previamente reportado este trabajo o uno similar? ¿Son los problemas o los enfoques de este trabajo de investigación completamente nuevos?

- Relevancia: importancia, utilidad o aplicabilidad de las ideas, métodos o técnicas, o de cualquier otro producto de investigación.

- Pertinencia: conveniencia, compatibilidad, congruencia y adecuación del producto de la investigación a las áreas y tópicos propios de la gestión de proyectos. Se puede resolver intentando dar respuesta al siguiente interrogante: ¿Quizás la investigación podría ser mejor presentada en otra área que cubra temas diferentes a la gestión de proyectos dentro de una ingeniaría en particular?

- Trascendencia: valor, impacto, no-trivialidad e importancia de las ideas, métodos, técnicas y producto de investigación generado en el trabajo. 
- Visibilidad: el producto de investigación cuenta con alguna publicación en revista o evento de importancia en el área pertinente a la gestión de proyectos de una ingeniería en particular.

\subsection{Valoración del informe de investigación}

- Presentación: organización adecuada del documento y del lenguaje usado en él. Claridad en lo que el autor del trabajo de investigación ha logrado. El documento debe ser redactado de tal forma que quienes no son expertos puedan comprender la contribución principal del trabajo de investigación y los métodos empleados para obtener dichos resultados.

- Contenido: el documento deja ver claramente el objeto de investigación, el planteamiento metodológico, la estructura del trabajo, el cuerpo teórico utilizado, el conocimiento y dominio de la literatura citada, la consistencia y rigor en la argumentación, la conexión entre la parte teórica y los aportes realizados, la conexión de las conclusiones con el cuerpo y objeto del trabajo de investigación.
- Referencias: las referencias bibliográficas y fuentes de información están adecuadas y unificadamente referenciadas. Las fuentes de información son relevantes o actualizadas.

- Síntesis: el cuerpo del documento es sintético y deja los aspectos secundarios como anexos.

- Redacción: el texto tiene correcta ortografía y adecuada y coherente redacción.

\subsection{Valoración de la defensa pública o socia- lización de resultados de investigación}

- Presentación: los recursos utilizados para la defensa pública son adecuados.

- Rigor expositivo: el autor manifiesta en todo momento dominio del tema y presenta de manera sistemática

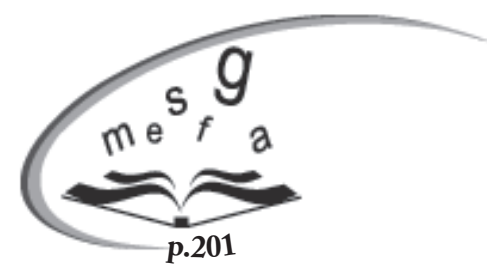
las ideas y los productos de investigación.

- Expresión adecuada: ausencia de ambigüedades, evasión de preguntas e imprecisiones en respuestas dadas. 


\section{CONCLUSIONES}

$\mathbf{L}$ a gestión de proyectos es una disciplina aplicable en gran variedad de profesiones y para el caso particular de este artículo, debe ser considerada como una línea de investigación dentro de los programas de ingeniería, buscando proyectos que aporten métodos, técnicas y herramientas en los diferentes procesos de gestión que se requieren en la construcción, operación o mantenimiento de productos de ingeniería.

Es necesario validar el procedimiento aquí propuesto dentro de los grupos de investigación en ingeniería, de manera que permita evidenciar la pertinencia de la gestión de proyectos en cualquiera de los procesos de gestión de proyectos de construcción, operación o mantenimiento de productos de ingeniería.
Cada proceso de gestión definido en la guía del $\mathrm{PMBOK}^{\circledR}$ aporta gran número de objetos de estudio que pueden ser creados, mejorados o adaptados mediante la actividad investigativa que se lleva a cabo en los programas de ingeniería.

Es posible desarrollar investigaciones que intenten realizar aportes generales en diferentes áreas de la gestión de proyectos; sin embargo, es conveniente acotar el alcance de la investigación, identificando el proceso de gestión con el que más relación tenga el producto de investigación. Esto facilita valorar la pertinencia y enfocar el estado del arte sobre un objeto de estudio claramente identificado.

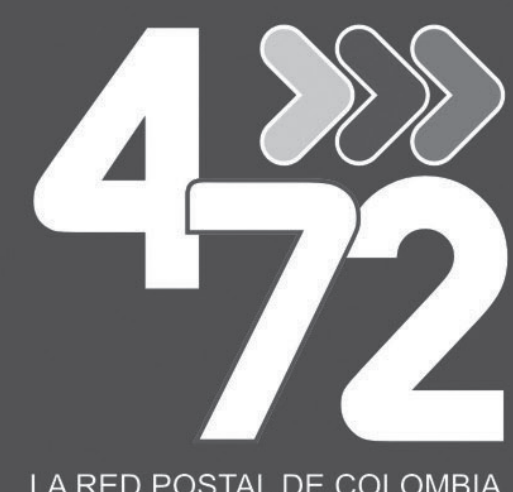

$w w w .4-72 \cdot c 0 m \cdot c 0$ 


\section{REFERENCIAS}

Galán, F. J. y Cañate, J. M. (2002). ¿Qué se entiende, en España, por investigación en ingeniería del software? Grupo ITÁLICA. Departamento de Lenguajes y Sistemas Informáticos. ETSI Informática. Universidad Sevilla.

IEEE (2004). Guide to the Software Engineering Body Of Knowledge. SWEBOK®. The Institute of Electrical and Electronics Engineers, Inc. IEEE Computer Society.

International Project Management Association (IPMA) (2009). NCB Bases para la competencia en dirección de proyectos, versión 3.1. España: Ed. AEIPRO.

Pinzón, W. y Ospina, A. (2006). Algunos elementos para la clasificación y evaluación de los proyectos de grado como trabajos de investigación en las facultades de ingeniería. STUDIOSITAS Revista de divulgación en ciencia, tecnología e innovación, 1(2), 15-22.

Pressman, R. S. (2010). Ingeniería del Software. Un enfoque práctico, séptima edición. Ciudad: McGraw-Hill.

Project Management Institute (PMI) (2009).Guía de los fundamentos para la dirección de proyectos. Guía del PMBOK®, cuarta edición.

Project Management Institute (PMI) (2013). Guide to the Project Management Body of Knowledge. PMBOK ${ }^{\circledR}$ Guide. Fifth Edition.

Project Management Institute (PMI) (2013a). Current PMI Standards Projects. Recuperado de http://www.pmi.org/en/ PMBOK-Guide-and-Standards/Standards-Current-PMI-Standards-Projects.aspx

Rojas Contreras, M., Esteban Villamizar, L. A. y Orjuela Duarte, Ailin (2011). Modelo de integración de las actividades de gestión de la guía del PMBOK ${ }^{\circledR}$, con las actividades de ingeniería, en proyectos de desarrollo de software. Revista Avances en Sistemas e Informática, 8(2), número de páginas.

Shaw, M. (1990). Prospects for an Engineering Discipline of Software. IEEE Software, pp. 15-24.

Shaw, M. (2002). What Makes Good Research in Software Engineering? European Joint Conference of Theory and Practice of Software (ETAPS 2002). International Journal on Software Tools for Technology Transfer, volumen, número y número de páginas. 